\title{
Confiabilidade da previsão da acuidade visual pós-operatória de catarata mediante medição da acuidade visual pré-operatória utilizando o retinômetro Heine Lambda 100
}

\author{
Reliability of predictable postoperative visual acuity of cataracts as \\ measured by Heine Lambda 100 retinometer preoperatively
}

\author{
Guilherme Novoa Colombo-Barboza ${ }^{1}$ \\ Wilson Takashi Hida ${ }^{2}$ \\ Arthur Van den Berg ${ }^{3}$ \\ Henrique Celso Duarte de Rezende Rocha ${ }^{4}$ \\ Gustavo Ricci Malavazzi ${ }^{5}$ \\ Luiz Roberto Colombo Barboza ${ }^{6}$ \\ Maria Margarida Colombo-Barboza ${ }^{7}$ \\ Marcello Novoa Colombo-Barboza ${ }^{8}$
}

\footnotetext{
Trabalho realizado no Departamento de Oftalmologia da Santa Casa de Misericórdia de São Paulo (SP) - Brasil.

Médico residente do Departamento de Oftalmologia da Irmandade Santa Casa de Misericórdia de São Paulo São Paulo (SP) - Brasil.

${ }^{2}$ Estagiário do Setor de Catarata da Irmandade Santa Casa de Misericórdia de São Paulo - São Paulo (SP) Brasil.

3 Médico residente do Departamento de Oftalmologia da Irmandade Santa Casa de Misericórdia de São Paulo São Paulo (SP) - Brasil.

${ }^{4}$ Médico residente do Departamento de Oftalmologia da Irmandade Santa Casa de Misericórdia de São Paulo São Paulo (SP) - Brasil.

Chefe do Setor de Catarata da Irmandade Santa Casa de Misericórdia de São Paulo - São Paulo (SP) - Brasil.

${ }^{6}$ Chefe de Oftalmologia da Faculdade de Medicina de Santos (UNILUS), Diretor Presidente do Hospital Oftalmológico Visão Laser - Santos (SP) - Brasil.

Chefe do Setor de Refração do Hospital Oftalmológico Visão Laser - Santos (SP) - Brasil.

${ }^{8}$ Estagiário do Setor de Córnea da Irmandade Santa Casa de Misericórdia de São Paulo - São Paulo (SP) - Brasil. Clínico Hospital Oftalmológico Visão Laser - Santos (SP) - Brasil.

Endereço para correspondência: Guilherme Novoa

Colombo-Barboza. Rua Jaguaribe, 102 - Apto. 142 - São

Paulo (SP) CEP 01224-001

E-mail: guilhermecolombo@hotmail.com

Recebido para publicação em 10.11 .2009

Última versão recebida em 26.05.2010

Aprovação em 22.06.2010

Nota Editorial: Depois de concluída a análise do artigo sob sigilo editorial e com a anuência do Dr. José Beniz Neto sobre a divulgação de seu nome como revisor, agradecemos sua participação neste processo.
}

\begin{tabular}{|l|}
\hline RESUMO \\
\hline Objetivo: Utilizaro retinômetro de Heine Lambda 100 para avaliara relação \\
da acuidade visual obtida no pré-operatório de cirurgia de catarata com a \\
acuidade visual obtida 3 meses no pós-operatório com correção óptica, \\
bem como, sua correlação com a classificação morfológica dominante da \\
catarata e com a intensidade da opacificação quando do tipo nuclear. \\
Métodos: Trata-se de um estudo prospectivo realizado no Hospital \\
Oftalmológico Visão Laser, em Santos, envolvendo 121 olhos de 70 \\
pacientes avaliados de abril ajulho 2009 , submetidos à cirurgia de catarata \\
sob a técnica de facoemulsificação com implante de lente intraocular. No \\
período pré-operatório, foi realizado o retinômetro de Heine sob midríase \\
e seu resultado foi comparado à melhor acuidade visual pós-operatória do \\
terceiro mês e correlacionado com a classificação morfológica da catarata, \\
quando do tipo nuclear, sendo denominado satisfatório aquele resultado \\
que não variou mais do que duas linhas na tabela de Snellen. Resultados: \\
Os resultados satisfatórios em nosso estudo foram de $86,78 \%$, apresentan- \\
do resultados de acuidade visual com retinômetro de Heine igual ao \\
resultado da acuidade visual pós-operatória em $34,7 \%$. A opacidade \\
predominantemente nuclearN1+tem um porcentual de acerto maior do que \\
N2+e N3+(50\%, 31,3\%e $26,7 \%$, respectivamente). Em relação ao total de \\
olhos, observamos um teste extremamente significante (p<0,0001). Con- \\
clusão: Oretinômetro de Heine hipoestimou ou manteve a acuidade visual \\
pós-operatória corrigida após 3 meses dos pacientes submetidos à \\
facectomia, na maioria dos casos. Ao correlacionar com a classificação \\
morfológica da catarata, observamos que, quanto maior a opacidade do \\
cristalino do tipo nuclear, maior a hipoestimação da acuidade visual. \\
\end{tabular}

Descritores: Catarata/fisiopatologia; Acuidade visual; Extração de catarata; Testes de visão/instrumentação; Técnicas de diagnóstico oftalmológico/instrumentação; Facoemulsificação; Implante de lente intraocular; Cuidados pré-operatórios; Cuidados pós-operatórios

\section{INTRODUÇÃ̃O}

A cirurgia de catarata evoluiu muito ao longo dos anos às custas de novos conceitos como indicação cirúrgica, tipos e materiais de lentes intraoculares, aparelhos de facoemulsificação e técnicas cirúrgicas ${ }^{(1)}$. 
As modificações no cristalino que ocorrem com o passar do tempo levam à diminuição da sensibilidade ao contraste ${ }^{(2)}$ e a qualidade da visão é ainda mais prejudicada quando se desenvolve catarata. Ao submeterem-se à facectomia, os pacientes têm expectativa de melhora da sua visão, com consequente melhora na qualidade de vida ${ }^{(3)}$. Na expectativa da melhora funcional da visão, vários exames pré-operatórios podem ser indicados, tais como o potencial de visão, interferômetro a laser, super pinhole, "potential acuity meter" (PAM), retinômetro de Heine (RH) entre outros. Desse modo, podemos prever, com certa margem de segurança, as chances de proporcionarmos boa acuidade visual ao paciente após o procedimento cirúrgico ${ }^{(4-8)}$.

O RH "Lambda 100" (Retinômetro de Heine, Herrsching, Germany) é um aparelho manual, portátil, de ampla mobilidade e fácil manuseio, por ser independente da lâmpada de fenda. Trata-se de um instrumento que tem como objetivo estimar a melhor acuidade visual corrigida opticamente, sem influência da opacificação dos meios, através da projeção de luz de xenônio na mácula, na forma de linhas paralelas de alto contraste e bordas bem definidas, com diferentes frequências espaciais ${ }^{(9)}$.

Conforme a literatura, podemos comparar o RH com o "potential acuity meter" (PAM), pois ambos têm a mesma função e mecanismo de ação ${ }^{(10-11)}$.

O objetivo deste trabalho foi comparar a acuidade visual obtida pelo retinômetro de Heine "Lambda 100" no período pré-operatório da cirurgia de catarata, com a acuidade visual corrigida opticamente após o terceiro mês da operação, bem como sua correlação com a classificação morfológica dominante da catarata e com a intensidade da opacificação, quando do tipo nuclear.

\section{MÉTODOS}

Trata-se de estudo prospectivo, aleatório, duplo-cego realizado no Hospital Oftalmológico Visão Laser, em Santos, envolvendo 121 olhos de 70 pacientes, sendo 39 (32\%) homens e 82 (68\%) mulheres, avaliados de abril a julho de 2009, submetidos à cirurgia de catarata, pelo mesmo cirurgião com a técnica de facoemulsificação e implante de lente intraocular.

O estudo foi aprovado pelo Comitê de Ética e Pesquisa do Hospital Visão Laser (Protocolo $n^{\circ}$ 7/09) e realizado após assinatura do Termo de Consentimento Livre e Esclarecido por todos os participantes.

\section{Seleção dos pacientes}

No período pré-operatório, foi realizado exame oftalmológico completo, composto por medida da melhor acuidade visual corrigida opticamente, tonometria de aplanação, biomicroscopia de segmento anterior, fundoscopia e biometria de imersão.

Uma vez obedecidos os critérios de inclusão e exclusão, foi então medida a acuidade visual com o RH, sob midríase medicamentosa (Figuras 1 e 2).

\section{Examples of various acuity grating patterns (visual angle $1^{\circ}$ )}
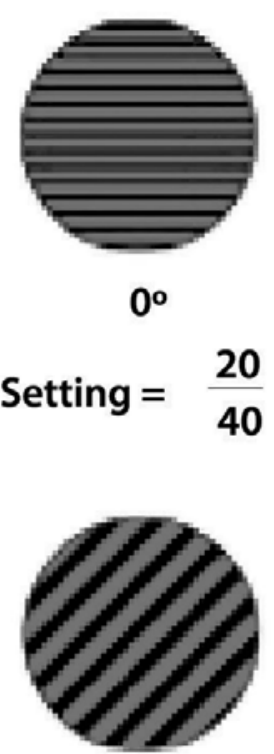

$45^{\circ}$
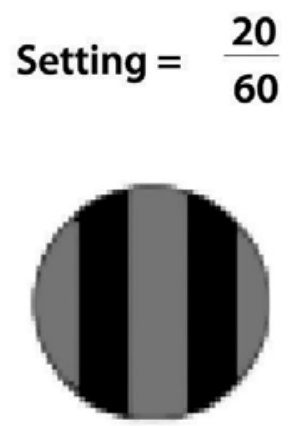

$90^{\circ}$

Setting $=\frac{20}{300}$

Figura 1 - Exame com o retinômetro de Heine (Lambda 100). Fonte: Manual do RH.

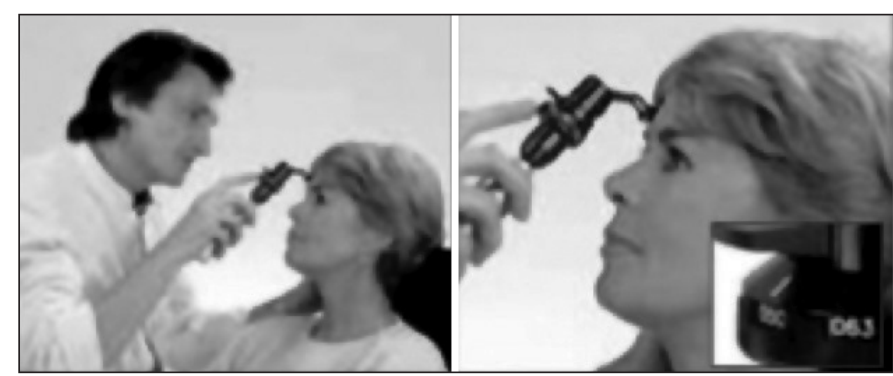

Figura 2 - Exame com o retinômetro de Heine (Lambda 100). Fonte: Manual do RH. 
246 Confiabilidade da previsão da acuidade visual pós-operatória de catarata mediante medição da acuidade visual pré-operatória utilizando o retinômetro Heine Lambda 100

\section{Critérios de inclusão}

- Pacientes com catarata bilateral;

- Idade 45 a 65 anos;

- Alfabetizados;

- Com visão corrigida opticamente no período pré-operatório melhor ou igual a $1,17 \log$ MAR;

- Astigmatismo corneal menor que 1,50 dioptria;

- Comprimento axial entre $22 \mathrm{~mm}$ e $25 \mathrm{~mm}$;

- Ausência de quaisquer outras doenças oculares, cirurgia ocular prévia ou uso de medicação hipotensora tópica.

\section{Critérios de exclusão}

- Complicações cirúrgicas ou pós-cirúrgicas que pudessem prejudicar a AV final;

- Cataratas densas, hipermaduras e subcapsular posterior 4+;

- Pacientes que não conseguiram realizar ou compreender o exame;

- Pacientes submetidos a capsulotomia posterior;

- Pacientes que não compareceram à consulta pós-operatória;

- Dúvidas quanto ao implante da LIO dentro do saco capsular ou descentração da LIO maior que $0,5 \mathrm{~mm}$, avaliada pelo exame à lâmpada de fenda;

- Pacientes com olho seco grave $\mathrm{e}^{(12)}$.

\section{Classificação morfológica da catarata}

Visando correlacionar os valores da acuidade visual obtida pelo retinômetro com a classificação morfológica da catarata, classificamo-la pela sua morfologia de forma subjetiva, sempre pelo mesmo examinador experiente, a fim de reduzir as variáveis de subjetividade entre examinadores, em nuclear (n1+ opacidade levemente amarelada até n4+ brunescente), cortical anterior, subcapsular posterior.

\section{Procedimento cirúrgico}

Todos os pacientes foram submetidos à facoemulsificação convencional com implante da lente intraocular Clariflex $^{\circledR}$ (AMO, Santa Ana, CA), pelo mesmo cirurgião experiente (W.T.H.).

\section{Seguimento pós-operatório}

Os pacientes foram submetidos a exame oftalmológico completo com um, sete, trinta e noventa dias pós-operatórios. Os dados da melhor acuidade visual obtidos no terceiro mês foram comparados aos da acuidade visual obtidos com o retinômetro Lambda e, em seguida, relacionados com a classificação morfológica da catarata.

Foram comparados os dados do RH do pré-operatório com o resultado visual de 3 meses pós-operatório sendo classificados em satisfatório (com variação de até 2 linhas na tabela de Snellen, com a melhor correção óptica) e insatisfatório (os demais).

Todas as avaliações da acuidade visual pós-operatória foram realizadas com a tabela de Snellen em pés (transforma- da em logMAR), monocularmente, com pupilas "não dilatadas", com e sem a melhor correção óptica.

\section{Análise estatística}

Para a comparação entre os dados pré e pós-operatórios, foram utilizados os testes " $t$ " de Student (para distribuição normal - gaussiana) e de Wilcoxon (para distribuição nãogaussiana).

Foi testada também a correlação de Spearman, entre os valores da acuidade visual do pré-operatório estimada pelo retinômetro de Heine e a acuidade visual pós-operatória (três meses com correção).

Foram utilizados os softwares Excel do pacote Microsoft Office e os softwares estatísticos GraphPad InStat 3 Versão 3.06 e SPSS 10.

\section{RESULTADOS}

Os resultados estão nas tabelas 1 a 4

Não observamos diferenças significantes entre os grupos quanto à idade, sexo e olho operado. Em relação ao total de olhos, observamos diferenças significantes $(p<0,0001)$ entre a acuidade visual pré-operatória e pós-operatória considerando todas as variedades de catarata, mostrando que provavelmente a acuidade visual (AV) pós-operatória deverá estar hipoestimada no pré-operatório com o RH.

Utilizando o teste da correlação de Spearman (Tabela 2), observamos forte correlação entre os valores pré e pós-operatórios ( $\mathrm{r}=0,713)$ de acuidade visual.

Observamos que o número de olhos com resultado satisfatório após 3 meses da cirurgia, em comparação com a acuidade visual obtida pré-operatoriamente mediante o RH foi de 105 $(86,78 \%)$ e os com resultados insatisfatórios foi de $16(13,22 \%)$.

\section{DISCUSS ÃO}

Os exames que estimam a acuidade visual pós-operatória constituem bom método preditivo, principalmente em cataratas ainda não muito densas ${ }^{(4-5)}$. Com este conhecimento, o cirurgião pode apresentar ao seu paciente uma expectativa mais real sobre os resultados visuais pós-operatórios.

O número de casos satisfatórios encontrados utilizando o RH no nosso estudo foi de $86,78 \%$, próximo aos $91 \%$ apresentados por alguns autores, utilizando o PAM, um estudo com 42 olhos e que não classificava o tipo da catarata.

Já quando comparado com o de outros autores que encontraram $67,7 \%$, num estudo bem próximo ao nosso, porém com 62 olhos, encontramos um valor superior.

Considerando-se 3 linhas de variação na tabela de Snellen, nosso estudo encontrou 95,6\%, enquanto que alguns autores descrevem $100 \%^{(13)}$.

Neste trabalho, o RH apresentou estimativas da acuidade visual iguais às encontradas pós-operatoriamente em 38,2\% 
Confiabilidade da previsão da acuidade visual pós-operatória de catarata mediante medição da acuidade visual pré-operatória utilizando o retinômetro Heine Lambda 100

Tabela 1. Relação entre a opacidade nuclear e a acuidade visual estimada no pré-operatório pelo retinômetro de Heine (Lambda 100), e 3 meses após a facectomia com implante de lente intraocular, pelo logMAR

\begin{tabular}{|c|c|c|c|c|}
\hline \multirow{2}{*}{$\begin{array}{l}\text { Subgrupos } \\
\text { Nuclear }\end{array}$} & \multirow[b]{2}{*}{ N (\%) } & logMAR pré & logMAR pós & \multirow[t]{2}{*}{ valor $p$} \\
\hline & & $\overline{\text { Mediana, Mínima e Máxima }}$ & $\overline{\text { Mediana, Mínima e Máxima }}$ & \\
\hline $\mathrm{n} 1+$ & $40(44,9 \%)$ & $0,17 / 0,00$ e 0,47 & $0,09 / 0,0$ e 0,47 & $<0,0001\left(^{*}\right)$ \\
\hline $\mathrm{n} 2+$ & $32(36,0 \%)$ & $0,32 / 0,09$ e 0,47 & $0,17 / 0,0$ e 0,47 & $0,0002\left(^{*}\right)$ \\
\hline n3+ & $15(16,9 \%)$ & $0,30 / 0,00$ e 0,87 & $0,17 / 0,0$ e 0,87 & $0,0186\left(^{*}\right)$ \\
\hline n4+ & $2(\quad 2,2 \%)$ & & & \\
\hline Total & $89(100,0 \%)$ & & & \\
\hline \multicolumn{5}{|c|}{$\begin{array}{l}\text { Fonte: Hospital Oftalmológico Visão Laser, Santos-SP, 14-09-2009 } \\
\text { n1+= nuclear +; n2+= nuclear ++; n3+= nuclear +++; n4+= nuclear ++++ } \\
\left(^{\star}\right) \text { Teste de Wilcoxon para amostras pareadas e distribuição não-gaussiana }\end{array}$} \\
\hline
\end{tabular}

\begin{tabular}{|c|c|c|c|c|}
\hline & \multirow[b]{2}{*}{ N (\%) } & logMAR pré & logMAR pós & \multirow[t]{2}{*}{ valor $p$} \\
\hline & & Mediana, Mínima e Máxima & Mediana, Mínima e Máxima & \\
\hline$n-c a$ & $14(11,6 \%)$ & $0,385 / 0,18$ e 1,17 & $0,18 / 0,0$ e 1,0 & $\left.0,0068 \quad{ }^{*}\right)$ \\
\hline n-scp & $9(7,4 \%)$ & $0,480 / 0,18$ e 1,18 & $0,48 / 0,18$ e 1,18 & $p=1$ \\
\hline n-ca-scp & $1(\quad 0,8 \%)$ & - & - & - \\
\hline$n$ & $89(73,6 \%)$ & $0,180 / 0,00$ e 0,87 & $0,09 / 0,00$ e 0,87 & $\left.<0,0001 \quad{ }^{*}\right)$ \\
\hline $\operatorname{scp}$ & $7(\quad 5,8 \%)$ & $0,276 \pm 0,120$ & $0,201 \pm 0,110$ & $0,0363\left({ }^{* *}\right)$ \\
\hline $\mathrm{ca}$ & $1(0,8 \%)$ & & & \\
\hline Total & $121(100,0 \%)$ & $0,300 / 0,00$ e 1,18 & $0,18 / 0,00$ e 1,18 & $<0,0001 \quad\left(^{*}\right)$ \\
\hline \multicolumn{5}{|c|}{$\begin{array}{l}\text { Fonte: Hospital Oftalmológico Visão Laser - Santos-SP, } 2009 \\
\mathrm{n}=\text { nuclear; } \mathrm{scp}=\text { subcapsular posterior; } \mathrm{ca}=\text { capsular anterior }\end{array}$} \\
\hline
\end{tabular}

\begin{tabular}{|c|c|c|c|c|}
\hline & \multirow[b]{2}{*}{$N \quad(\%)$} & \multirow{2}{*}{$\frac{\text { Hiperestimativa }}{\mathrm{N}(\%)}$} & Igual & Hipoestimativa \\
\hline & & & $\begin{array}{l}\mathrm{N} \quad(\%) \\
\end{array}$ & $N \quad(\%)$ \\
\hline n-ca & $14(11,6 \%)$ & $1(7,1 \%)$ & $3(21,4 \%)$ & $10(71,4 \%)$ \\
\hline n-scp & $9(7,4 \%)$ & $0(0,0 \%)$ & $1(11,1 \%)$ & $8(88,9 \%)$ \\
\hline n-ca-scp & $1(0,8 \%)$ & $0(0,0 \%)$ & $0(\quad 0,0 \%)$ & $1(100,0 \%)$ \\
\hline$n$ & $89(73,6 \%)$ & $5(5,6 \%)$ & $34(38,2 \%)$ & $50(56,2 \%)$ \\
\hline $\operatorname{scp}$ & $7(5,8 \%)$ & $0(0,0 \%)$ & $3(42,9 \%)$ & $4(57,1 \%)$ \\
\hline ca & $1(0,8 \%)$ & $0(0,0 \%)$ & $1(100,0 \%)$ & $0,0 \%)$ \\
\hline Total & $121(100,0 \%)$ & $6(5,0 \%)$ & $42(34,7 \%)$ & 73( \\
\hline
\end{tabular}

Tabela 4. Relação entre a opacidade nuclear e a AV pós-cirúrgica

\begin{tabular}{|c|c|c|c|c|}
\hline \multirow{2}{*}{$\begin{array}{l}\text { Subgrupos } \\
\text { Nuclear }\end{array}$} & \multirow[b]{2}{*}{$N \quad(\%)$} & \multirow{2}{*}{$\frac{\text { Hiperestimativa }}{\mathrm{N}(\%)}$} & Igual & Hipoestimativa \\
\hline & & & $(\%)$ & $(\%)$ \\
\hline $\mathrm{n} 1+$ & $40(44,9 \%)$ & $2(5,00 \%)$ & $20(50,0 \%)$ & $18(45,00 \%)$ \\
\hline n2+ & $32(36,0 \%)$ & $2(6,30 \%)$ & $10(31,3 \%)$ & $20(62,50 \%)$ \\
\hline n3+ & $15(16,9 \%)$ & $1(6,70 \%)$ & $4(26,7 \%)$ & $10(66,70 \%)$ \\
\hline n4+ & $2(\quad 2,2 \%)$ & & & \\
\hline Total & $89(100,0 \%)$ & $5 \quad(5,62 \%)$ & $34(38,2 \%)$ & $48(53,94 \%)$ \\
\hline
\end{tabular}


248 Confiabilidade da previsão da acuidade visual pós-operatória de catarata mediante medição da acuidade visual pré-operatória utilizando o retinômetro Heine Lambda 100

das cataratas nucleares, contra $21 \%$ no PAM como descrito na literatura ${ }^{(4)}$.

Quando avaliamos separadamente cada tipo de opacidade nuclear, o porcentual de acerto diminuiu com o aumento da intensidade, tais resultados assemelham-se aos da literatura pesquisada $^{(4)}$

A opacidade predominantemente nuclear $\mathrm{n} 1+$ tem porcentual de acerto maior do que $n 2+$ e $n 3+(50 \%, 31,3 \%$ e $26,7 \%$, respectivamente) fato que se assemelha aos resultados na literatura. Em nosso estudo, não incluímos os pacientes com doenças oculares, considerando apenas os normais. Desse modo, podemos num segundo momento aplicar o $\mathrm{RH}$ em pacientes com catarata associada a doenças retínicas como degenerações maculares, retinopatia diabética ou hipertensiva, entre outras.

Para analisamos a associação dos outros tipos morfológicos das cataratas com a acuidade visual pré e pós-operatória estimada pelo $\mathrm{RH}$, nos tipos subcapsular posterior e/ou cortical anterior, devemos constituir uma amostragem maior. Neste trabalho analisamos apenas o tipo nuclear por ser o mais comum.

Este exame deve ser um parâmetro a ser somado ao quadro clínico do paciente, tendo em vista a variação apresentada em relação à morfologia dominante da catarata e à sua hipoestimação na maioria dos casos estudados ${ }^{(14-15)}$.

\section{CONCLUSÃO}

Concluindo, o retinômetro de Heine "Lambda 100" hipoestimou ou manteve o prognóstico da acuidade visual pós-operatória dos pacientes submetidos à facectomia, na maioria dos casos. Ao correlacionar com a classificação morfológica da catarata, observamos que, quanto maior a opacidade nuclear do cristalino, maior a hipoestimação da acuidade visual.

\section{AGRADECIMENTOS}

Dr. Wilmar Silvino, Dr. Geraldo Vicente Almeida, Dra Maria Cristina Nishiwaki-Dantas, Dr. Paulo Elias C. Dantas, Dr. Carlos Sousa-Dias, Marina Colombo Barboza M. Francisco, Dra. Fernanda Daroz Paulo e Isalinda Gonçalves Novoa pelas inestimáveis colaborações e disponibilidade em dividir seus conhecimentos conosco.

\section{ABSTRACT}

Purpose: To assess the relationship between potential visual acuity obtained before cataract surgery using Heine Lambda 100 retinometer with best corrected visual acuity 3 months postoperatively, as well as its correlation with the morphological pattern of the dominant cataract and the intensity of nuclear opacification. Methods: Prospective study executed in the Ophthalmology Hospital Laser Vision in Santos of 121 eyes of 70 patients who underwent cataract surgery (phacoemulsification with intraocular lens implantation), from April to July, 2009. In the preoperative period, Heine retinometer test was performed under mydriasis and its results were compared to postoperative best corrected visual acuity three months after surgery and correlated with the morphological classification of cataracts, being considered a satisfactory result those who did not vary more than two lines in Snellen chart. Results: The satisfactory cases found in our study were $86.78 \%$, with results of visual acuity with Heine retinometer equal to the postoperative visual acuity in $34.7 \%$ of the cases. Predominant nuclear opacity $\mathrm{N} 1+$ has a higher reliability than $\mathrm{N} 2+$ and $\mathrm{N} 3+(50 \%, 31.3 \%$ and $26.7 \%$, respectively). Regarding all studied eyes, statistical significance was noted $(\mathrm{p}<0.0001)$. Conclusion: In most cases Heine retinometer underestimated or maintained best corrected visual acuity 3 months postoperatively in patients who underwent cataract surgery. With respect to the morphological classification of cataracts, the higher the opacity of the nuclear lens, the greater the visual acuity underestimation.

Keywords: Cataract/physiopathology; Visual acuity; Cataract extraction; Vision tests/instrumentation; Diagnostic techniques ophthalmological; Phacoemulsification; Lens implantation, intraocular; Preoperative care; Postoperative care

\section{REFERÊNCIA}

1. Obuchowska I, Mariak Z. [Sir Harold Ridley-the creator of modern cataract surgery]. Klin Oczna. 2005;107(4-6):382-4. Polish.

2. Alio JL, Schimchak P, Negri HP, Montes-Mico R. Crystalline lens optical dysfunction through aging. Ophthalmology. 2005;112(11):2022-9.

3. Rawer R, Stork W, Spraul CW, Lingenfelder C. Imaging quality of intraocular lenses. J Cataract Refract Surg. 2005:31(8):1618-31. Comment in: J Cataract Refract Surg. 2006;32(4):545-6; author reply 546.

4. Chiacchio BB, Sato RM, Siqueira RB, Marques FF. [Fidelity of the potential acuity meter in the postoperative visual acuity of cataract surgery]. Arq Bras Oftalmol. 2008;71(6):805-8. Portuguese.

5. Odom JV, Chao GM, Weinstein GW. Preoperative prediction of postoperative visual acuity in patients with cataracts: a quantitative review. Doc Ophthalmol. 1988;70(1):5-17.

6. Barrett BT, Davison PA, Eustace P. Clinical comparison of three techniques for evaluating visual function behind cataract. Eye (Lond). 1995;9(Pt 6):722-7.

7. Datiles MB, Edwards PA, Kaiser-Kupfer MI, McCain L, Podgor M. A comparative study between the PAM and the laser interferometer in cataracts. Graefes Arch Clin Exp Ophthalmol. 1987;225(6):457-60.

8. Smiddy WE, Radulovic D, Yeo JH, Stark WJ, Maumenee AE. Potential acuity meter for predicting visual acuity after Nd:YAG posterior capsulotomy. Ophthalmology. 1986;93(3):397-400.

9. Tharp A, Cantor L, Yung CW, Shoemaker J. Prospective comparison of the Heine retinometer with the Mentor Guyton-Minkowski potential acuity meter for the assessment of potential visual acuity before cataract surgery. Ophthalmic Surg. 1994;25(9):576-9. Comment in: Ophthalmic Surg. 1994;25(9):657.

10. Lasa MS, Datiles MB, $3^{\text {rd }}$, Freidlin V. Potential vision tests in patients with cataracts. Ophthalmology. 1995;102(7):1007-11.

11. Severin TD, Severin SL. A clinical evaluation of the potential acuity meter in 210 cases. Ann Ophthalmol. 1988;20(10):373-5.

12. Barboza MNC, Barboza GNC, Melo GM, Sato É, Dantas MCN, Dantas PEC, et al. Correlação entre sinais e sintomas de olho seco em pacientes portadores da síndrome de Sjögren. Arq Bras Oftalmol. 2008;71(4):547-52.

13. Minkowski JS, Palese M, Guyton DL. Potential acuity meter using a minute aerial pinhole aperture. Ophthalmology. 1983;90(11):1360-8.

14. Melki SA, Safar A, Martin J, Ivanova A, Adi M. Potential acuity pinhole: a 
simple method to measure potential visual acuity in patients with cataracts, comparison to potential acuity meter. Ophthalmology. 1999;106(7):1262-7.

Comment in: Ophthalmology. 2000;107(4):623-4.
15. Reis FAC, Cohen R, Neufeld CR, Dias ACT, Pereira DS. Visual prognosis for lens extraction: Heine retinometer and multiple pinhole. Arq Bras Oftalmol. 2004;67(5):759-62.

+口in

\section{Jornada Anual do Conselho Brasileiro de Ortóptica}

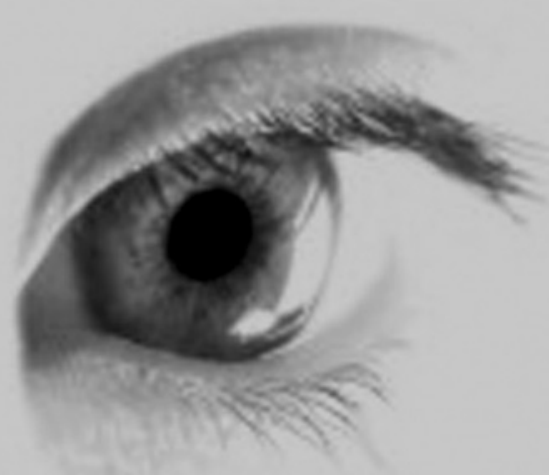

\section{4 de agosto de 2010} Hotel Mercure - Previllege *....: São Paulo - SP

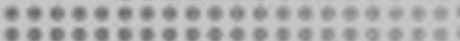

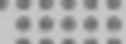

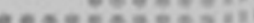
(1)

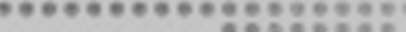

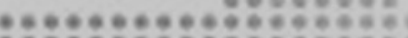

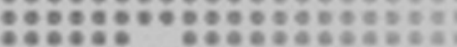

$0: 0: 0$

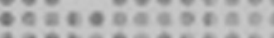

Informações:

e-mail: secretaria@cbort.com.br site: www.cbort.com.br 